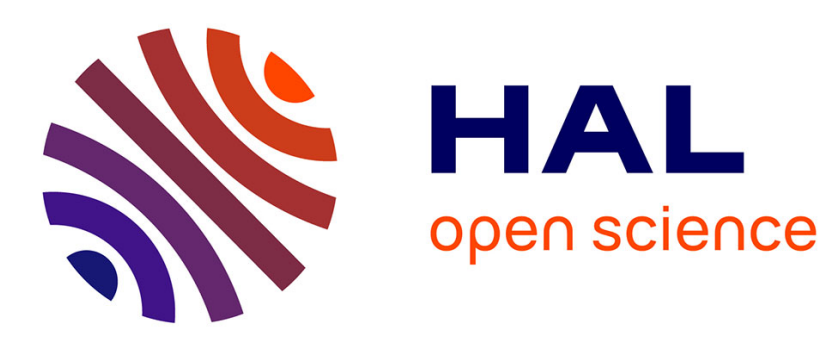

\title{
Charge density wave in graphene: magnetic-field-induced Peierls instability
}

Jean-Noël Fuchs, Pascal Lederer

\section{To cite this version:}

Jean-Noël Fuchs, Pascal Lederer. Charge density wave in graphene: magnetic-field-induced Peierls instability. The European Physical Journal. Special Topics, 2007, 148, pp.151-158. 10.1140/epjst/e2007-00234-4 . hal-00120464

\section{HAL Id: hal-00120464 \\ https://hal.science/hal-00120464}

Submitted on 15 Dec 2006

HAL is a multi-disciplinary open access archive for the deposit and dissemination of scientific research documents, whether they are published or not. The documents may come from teaching and research institutions in France or abroad, or from public or private research centers.
L'archive ouverte pluridisciplinaire HAL, est destinée au dépôt et à la diffusion de documents scientifiques de niveau recherche, publiés ou non, émanant des établissements d'enseignement et de recherche français ou étrangers, des laboratoires publics ou privés. 
EPJ manuscript No.

(will be inserted by the editor)

\title{
Charge density wave in graphene: magnetic-field-induced Peierls instability
}

\author{
Jean-Noël Fuchs ${ }^{1, a}$ and Pascal Lederer ${ }^{1}$ \\ Laboratoire de Physique des Solides, Univ. Paris-Sud, CNRS, UMR 8502, F-91405 Orsay Cedex, France
}

\begin{abstract}
We suggest that a magnetic-field-induced Peierls instability accounts for the recent experiment of Zhang et al. in which unexpected quantum Hall plateaus were observed at high magnetic fields in graphene on a substrate. This Peierls instability leads to an out-of-plane lattice distortion resulting in a charge density wave (CDW) on sublattices A and B of the graphene honeycomb lattice. We also discuss alternative microscopic scenarios proposed in the literature and leading to a similar CDW ground state in graphene.
\end{abstract}

\section{Introduction}

The recent interest in graphene was triggered by the observation of an unusual "relativistic" quantum Hall $(\mathrm{QH})$ effect by two groups [1,2]. This effect is a direct consequence of the peculiar density of states of graphene close to half-filling [3]. Shortly after these pioneering experiments, Zhang et al. [4] explored the regime of even larger magnetic fields, up to $45 \mathrm{~T}$, and observed extra plateaus in the Hall conductivity $\sigma_{x y}$. In contrast to the relativistic quantum Hall effect observed at lower magnetic fields, these extra plateaus cannot be explained by considering non-interacting electrons. In the present paper, we review the recent proposal of a magneticfield-induced Peierls instability for graphene on a substrate [5], which gives an explanation to the extra quantum Hall plateaus observed at high magnetic field. The Peierls instability leads to an out-of-plane lattice distortion resulting in a charge density wave (CDW) on sublattices A and $\mathrm{B}$ of the graphene honeycomb lattice. Other mechanisms leading to such a CDW ground state in graphene have been proposed in the literature and are discussed in the present paper [6, 7, 8, 9]. The originality of the magnetic-field-induced Peierls instability scenario [5] is to rely on electron-phonon coupling via the substrate on which the graphene flake lies, rather than on Coulomb interactions between electrons [6, [7, 8, 9]

\section{Quantum Hall effect in graphene: non-interacting electrons}

The graphene crystal is a honeycomb lattice of carbon atoms: a two-dimensional triangular Bravais lattice with a basis of two atoms, usually referred to as A and B. The distance between nearest neighbor atoms is $a=0.14 \mathrm{~nm}$ and the lattice constant is $a \sqrt{3}$. Experimentally, graphene sheets of area $\mathcal{A} \sim(3-10 \mu \mathrm{m})^{2}$ are deposited on amorphous $\mathrm{SiO}_{2} / \mathrm{Si}$ substrate. Applying a gate voltage $V_{g}$ via the substrate allows one to control the electronic filling of the graphene bands, which amounts to doping. The number of induced electronic charges is given by $N_{c}=V_{g} C_{g} / e$ where the capacitance per unit area can be estimated as $C_{g} / \mathcal{A} \approx \epsilon / d \approx 1.2 \times 10^{-4} \mathrm{~F} / \mathrm{m}^{2}$, where $-e<0$ is the electron charge, $\epsilon=\epsilon_{0} \epsilon_{r}$ (with $\epsilon_{r} \approx 4$ ) is the silicon oxide dielectric constant and its thickness is $d \sim 300 \mathrm{~nm}[1$, 2].

\footnotetext{
a e-mail: fuchs@lps.u-psud.fr
} 
The electronic properties of graphene are described by a standard nearest-neighbor tightbinding model [10 with hopping amplitude $t \approx 3 \mathrm{eV}[11]$ between $2 p_{z}$ carbon orbitals. Each carbon atom contributes one conduction electron. There are $2 N_{p}$ electrons in the sample under zero gate voltage, where $N_{p}$ is the number of unit cells in the sample. The first Brillouin zone is hexagonal. Among its six corners, only two are inequivalent and called $K$ and $K^{\prime}$. We choose $\mathbf{K}=4 \pi /(3 \sqrt{3} a) \mathbf{u}_{x}$ and $\mathbf{K}^{\prime}=-\mathbf{K}$. The resulting band structure features the merging of the conduction and valence band at precisely these two points: graphene is a two-valley ( $K$ and $\left.K^{\prime}\right)$ zero-gap semiconductor. Near these so-called Dirac points, the electrons behave as charged massless chiral fermions and the valence and conduction bands have the shape of a diabolo. The relativistic-like dispersion relation of electrons is $\varepsilon_{k}= \pm \hbar v_{F}|\mathbf{k}|$, with a Fermi velocity $v_{F}=3 a t / 2 \hbar \approx 10^{6} \mathrm{~m} / \mathrm{s}$ about 300 times smaller than the velocity of light $c$. This results in a linear density of states close to the Dirac point. In undoped graphene $\left(V_{g}=0\right)$, the valence band is filled and the conduction band is empty: the chemical potential is right at the Dirac points. Changing the gate voltage from zero allows one to fill the conduction band or empty the valence band.

In order to discuss the quantum Hall effect in graphene, we briefly review the Landau problem of a graphene electron in a perpendicular magnetic field [12]. The magnetic field is assumed weak enough to neglect any magnetic flux commensuration effect (for a study of the Hofstadter butterfly in graphene, see [13 ) and we use the continuum approximation in which the tight-binding model reduces to the two-dimensional massless Dirac Hamiltonian. The Landau levels (LL) are

$$
\varepsilon_{n}=\operatorname{sgn}(n) \sqrt{|n|} \hbar \omega_{c}
$$

where the "cyclotron" frequency is $\omega_{c}=v_{F} \sqrt{2 e B_{\perp} / \hbar}$, and the LL index $n$ is an integer. The unusual LL structure with a square root dependence in the perpendicular magnetic field is a direct consequence of the linear density of states close to the Dirac point. Each LL is $4 N_{\phi}$ times degenerate, where the number of flux quanta across the sample $N_{\phi}=e B_{\perp} \mathcal{A} /(2 \pi \hbar)$ gives the orbital degeneracy and the factor 4 accounts for twofold spin $1 / 2$ and twofold valley degeneracy.

Let $\nu=N_{c} / N_{\phi}=C_{g} V_{g} / e N_{\phi}$ be the filling factor. When the gate voltage is zero, so is the filling factor and the central Landau level (CLL, $n=0$ ) is half-filled as a result of particlehole symmetry. In addition, each LL is $4 N_{\phi}$ times degenerate. These two facts alone show that a plateau at $\nu e^{2} / h$ occurs in the Hall conductivity $\sigma_{x y}$ each time the filling factor is close to $\operatorname{sgn}(n)(4|n|+2)= \pm 2 ; \pm 6 ; \pm 10 ; \ldots$ In order to have extra quantum Hall plateaus, one has to provide mechanisms for lifting the spin and valley degeneracy. In the absence of interactions between electrons, the only such mechanism is the Zeeman effect, which lifts the spin degeneracy, but does not affect the twofold valley degeneracy. The Zeeman effect, which depends on the total magnetic field $B_{\text {tot }}$, splits each LL and leads to a gap $\Delta_{Z}=g^{*} \mu_{B} B_{\text {tot }}$, where $\mu_{B}=e \hbar / 2 m$ is the Bohr magneton, $m$ is the bare electron mass, and $g^{*} \approx 2$ is the effective $g$-factor [4]. If the Zeeman gap is larger than the LL width, one expects extra quantum Hall plateaus at $\nu=4 n=0 ; \pm 4 ; \pm 8 ; \ldots$ Therefore in the absence of interactions between electrons, the only expected quantum Hall plateaus should occur at every even filling factor $\nu=2 n$. In the experiment of Zhang et al., plateaus at $\nu= \pm 1$ were observed at large magnetic field, suggesting that interactions between electrons play a role [4]. However, none of the other odd plateaus $\nu= \pm 3 ; \pm 5 ; \ldots$ were observed. It is very simple to understand these facts if one assumes that the electrons have different on-site energies on the two carbon atoms in the unit cell, just as for boron nitride, which also has a honeycomb structure [14. In that case the Dirac electrons become massive [14 and the LL are almost unchanged except for the CLL which is not valley degenerate anymore [15]: this is enough to understand the filling factors at which $\mathrm{QH}$ plateaus were observed. It now remains to find a microscopic mechanism leading to the generation of this mass. In the following section, we show that in the presence of a magnetic field phonon-mediated (indirect) interactions between electrons lead to a CDW ground state. The latter features different electronic charge densities on the two sublattices providing different on-site energies for $\mathrm{A}$ and $\mathrm{B}$ carbon atoms, and therefore leading to a mass for the Dirac electrons. 


\section{Magnetic-field-induced Peierls instability}

Undoped graphene is a very peculiar two-dimensional metal: it has a Fermi surface consisting of only two points $\left(\mathbf{K}, \mathbf{K}^{\prime}\right)$, which means that its density of states at the Fermi energy is vanishingly small. Such a Fermi surface features perfect nesting at wavevector $\mathbf{Q}=0$ and $\mathbf{K}$, as testified by the Kohn anomalies found in the spectrum of its optical in-plane phonons [16. This means that graphene is a quite unstable metal with respect to charge density modulations at wavevector $\mathbf{Q}$ and therefore, although two-dimensional, a good candidate for a Peierls instability [17]. Electrons in free-standing graphene are strongly coupled to the in-plane optical phonons but are not coupled to the out-of-plane ZO phonon [16]. Such a coupling is also absent in graphite because each graphene layer is in a symmetric environment with respect to its neighboring layers. However for graphene on a substrate, this is not the case: as the substrate (amorphous $\mathrm{SiO}_{2}$ ) is different from the superstrate (air), the coupling of electrons to the $\mathrm{ZO}$ phonons is not forbidden by symmetry. Such an electron-phonon coupling is actually provided by the interactions of the graphene sheet with the substrate, as we show below. The ZO phonons correspond to an out-of-plane vibration where the A and B atoms in the unit cell move out of phase. If this vibration is frozen-in, every second carbon atom is closer to the substrate than its partner in the unit cell: the honeycomb lattice is no longer flat but crinkled as an egg box. In that case, the $\mathrm{A}$ and $\mathrm{B}$ carbon atoms do not have the same on-site energy anymore, which means that the Dirac electrons have acquired a finite mass. In the following, we quantitatively develop this idea.

We now consider a spontaneous out-of-plane lattice distortion which - in the presence of the substrate - breaks the inversion symmetry of the honeycomb lattice and provides a mechanism for lifting the valley degeneracy in the CLL. Assume that the A (resp. B) sublattice moves away (resp. towards) the substrate by a distance $\eta$. Electrons are still described by a honeycomb nearest-neighbor tight-binding model, however the two atoms in the basis now have different on-site energies. The energy on atom $\mathrm{A} / \mathrm{B}$ is called $\pm M$. Close to the Dirac points and including the Zeeman effect, the LL read [15]

$$
\begin{aligned}
& \varepsilon_{n, \sigma, \alpha}=\operatorname{sgn}(n) \sqrt{M^{2}+2 \hbar v_{F}^{2} e B_{\perp}|n|}+\frac{g^{*}}{2} \mu_{B} B_{\text {tot }} \sigma \quad \text { if } \quad n \neq 0 \\
& \varepsilon_{0, \sigma, \alpha}=\alpha M+\frac{g^{*}}{2} \mu_{B} B_{\text {tot }} \sigma \quad \text { if } \quad n=0,
\end{aligned}
$$

where $\alpha= \pm 1$ is the valley index corresponding to the Dirac points $\alpha \mathbf{K}$, and $\sigma= \pm 1$ is the spin projection along the magnetic field direction. In terms of the low-energy effective theory, the distortion means that the Dirac electrons spontaneously acquire a finite mass $M / v_{F}^{2}$. The onsite energy difference lifts the valley degeneracy for the CLL only, with a valley gap $\Delta_{v}=2 M$. Note that the effect of a nonzero on-site energy $M$ on each $n \neq 0$ LL is very weak, of order $M^{2} / \hbar v_{F}^{2} e B_{\perp} \ll 1$ for a typical magnetic field. The reason is that in every LL, apart from the CLL, each quantum state - independently of its valley index - has an equal weight on the two sublattices. This is, however, not the case in the CLL: electrons at the $K$ (resp. $K^{\prime}$ ) point only reside on the $A$ (resp. $B$ ) sublattice. This explains why breaking the inversion symmetry of the honeycomb lattice (by introducing different on-site energies on the two sublattices) lifts the valley degeneracy of the CLL only.

Such a lattice distortion spontaneously occurs because it lowers the total energy, just as in Peierls's mechanism [17] except for the magnetic field playing an essential role here and for the crystal being two rather than one dimensional. Assume that the last partially filled LL is $n=0$ (i.e. the gate voltage $V_{g}$ is such that $|\nu| \leq 2$ ). We show that in this case it is always favorable to slightly distort the lattice provided there is a perpendicular magnetic field ${ }^{1}$. The distortion lowers the electronic energy. This energy lowering comes both from the CLL, which gives an essential contribution, and also from all the $n<0 \mathrm{LL}$, which contribute in a less important way as we explain below. There are $(2+\nu) N_{\phi}$ electrons in the CLL. They contribute an energy

\footnotetext{
${ }^{1}$ When $|\nu|>2$, there is no energy gained by distortion. The distortion only occurs for $\nu$ close to zero.
} 
gain

$$
E_{n=0}=-N_{\phi}(2-|\nu|) M
$$

because when $\nu<0$, all $(2+\nu) N_{\phi}$ electrons gain each an energy $M$ and when $\nu>0,2 N_{\phi}$ electrons gain each an energy $M$ but the remaining $\nu N_{\phi}$ electrons loose each an energy $M$. This energy gain depends on the magnetic field through $N_{\phi}$. In addition, the energy gain is linear in the out-of-plane distortion $\eta$ because the on-site energy is proportional to the distortion, as we discuss below: $M=D \eta$, where $D$ is a proportionality constant, akin to a deformation potential 2. The other $2\left(N_{p}-N_{\phi}\right)$ electrons that fill the $n<0 \mathrm{LLs}$, also contribute to the energy lowering. Each of them gains a small energy compared to what an $n=0$ electron gains, as discussed in the preceding paragraph, but as there are many more of them, about $2\left(N_{p}-N_{\phi}\right) \approx 2 N_{p}$, we cannot neglect their contribution. In the tight-binding model, we find

$$
E_{n<0}=-\gamma \frac{N_{p} a}{\hbar v_{F}} M^{2}
$$

where the numerical factor $\gamma \approx 0.67$. This energy gain is quadratic in the distortion, and therefore smaller than $E_{n=0}$ at small distortion, and independent of the magnetic field. Actually, this term represents the full electronic energy gain for a lattice distortion under zero magnetic field. The distortion costs an elastic energy

$$
E_{\text {elastic }}=N_{p} G \eta^{2}
$$

where the out-of-plane distortion is assumed to be small $\eta \ll a$ and $G$ is an elastic constant, which - in the adiabatic approximation - is related to the ZO phonon frequency at $\mathbf{q}=0$ by $\omega_{0}^{2} \approx 4 G / m_{c}$, where $m_{c}$ is the mass of a carbon atom and $\omega_{0} / 2 \pi$ is the phonon frequency at the $\Gamma$ point. From the measured frequency $\omega_{0} / 2 \pi c \sim 800 \mathrm{~cm}^{-1}$ of the graphite out-of-plane optical phonon 18], we obtain $G a^{2} \approx m_{c} \omega_{0}^{2} a^{2} / 4 \sim 14 \mathrm{eV}$.

The total energy is therefore

$$
E_{\mathrm{tot}}=E_{n=0}+E_{n<0}+E_{\text {elastic }}=-N_{\phi}(2-|\nu|) D \eta-\gamma \frac{N_{p} a}{\hbar v_{F}} D^{2} \eta^{2}+N_{p} G \eta^{2}
$$

The most remarkable difference in this expression with respect to the usual Peierls case is the presence of an energy gain linear in the lattice distortion $\eta$ rather than proportional to $\eta^{2} \ln \eta$ [19]. We note that the phonon mediated (indirect) electron-electron interaction is $g_{\text {el.-el. }}^{\text {eff. }} \sim$ $g_{\text {el.-ph. }}^{2} /\left(\hbar \omega_{0}\right) \sim D^{2} / G$. The dimensionless electron-electron coupling constant ${ }^{3} \lambda$ is therefore of order $g_{\text {el.-el. }}^{\text {eff. }} / t$. Comparing the kinetic energy gain (in the absence of a magnetic field) and the elastic cost in equation (7) shows that it is $\lambda=2 \gamma D^{2} /(3 G t)$.

As $E_{n<0}$ and $E_{\text {elastic }}$ are both quadratic in the lattice distortion, we introduce a renormalized elastic constant $G^{\prime}=G-\gamma a D^{2} / \hbar v_{F}$ and write an effective elastic energy:

$$
E_{\text {elastic }}+E_{n<0}=N_{p} G^{\prime} \eta^{2} .
$$

The effect of the $n<0$ electrons is to reduce the lattice stiffness and therefore to enhance the distortion. We take it as an experimental fact that there is no spontaneous out-of-plane distortion in the absence of a perpendicular magnetic field, see also [11, which means that $G^{\prime}>0$. This means that the Peierls instability does not occur in the absence of a magnetic field. For the ZO phonon, it means that - in the adiabatic approximation - its frequency is renormalized by the coupling to the electrons as $\omega^{2} \approx \omega_{0}^{2}+4\left(G^{\prime}-G\right) / m_{c}=\omega_{0}^{2}(1-\lambda)$. Measuring the ZO phonon mode in graphene on substrate would directly give access to $G^{\prime}$,

\footnotetext{
2 The corresponding electron-phonon coupling constant $g_{\text {el.-ph. }}$ that would appear in a Fröhlich-type Hamiltonian is $g_{\text {el.-ph. }} \sim D \sqrt{\hbar /\left(m_{c} \omega_{0}\right)}$, where $m_{c}$ is the mass of a carbon atom.

3 The quantity $\lambda$ is commonly known as the dimensionless electron-phonon coupling constant, e.g. in the context of the BCS theory of superconductivity, eventhough it is actually a dimensionless (phononmediated) electron-electron coupling constant [19].
} 
and therefore provide an independent determination of the constant $D$ through the equation $D=\sqrt{\left(G-G^{\prime}\right) \hbar v_{F} / \gamma a}$.

In order to obtain the ground state - within our mean-field approach - we minimize the total energy $E_{\text {tot }}$, see equation (7), as a function of the distortion $\eta$ and get

$$
\eta=\frac{N_{\phi}}{N_{p}} \frac{2-|\nu|}{2} \frac{D}{G^{\prime}},
$$

from which the valley splitting follows as

$$
\Delta_{v}=\frac{N_{\phi}}{N_{p}}(2-|\nu|) \frac{D^{2}}{G^{\prime}} \propto B_{\perp},
$$

and the condensation energy is $E_{\text {tot }}=-(2-|\nu|) N_{\phi} D \eta / 2$. Actually in the CLL, and only in that LL, the valley index is the same as the sublattice index. Therefore if at $\nu \approx 0$ all electrons in the CLL are in one valley, they are also only on one of the two sublattices: the ground state can therefore be described as a commensurate A-B (or site centered) CDW. The charge density oscillation is quite small however: $\left|N_{A}-N_{B}\right| / N_{p} \approx N_{\phi} / N_{p} \sim\left(a / l_{B}\right)^{2} \ll 1$, where $l_{B}=\sqrt{\hbar /\left(e B_{\perp}\right)}$ is the magnetic length.

The usual Peierls instability, which results in a gap opening at the Fermi energy in the electronic spectrum, is accompanied by a softening of the coupled phonon mode 19. Approaching the instability from the metallic side, the phonon mode softens and goes to zero precisely at the transition - a phenomenon known as a giant Kohn anomaly [19]. In order to see this effect in the case of the magnetic field induced Peierls instability where the driving parameter is the magnetic field rather than the temperature, we consider broadened LL. Broadening is due to disorder and slightly modifies the preceding calculation for lattice distortion at weak magnetic field, when the valley splitting $\Delta_{v}$ is smaller than the LL width $\Delta_{\text {imp. }}$. For example, for rectangular LL - the density of states being $4 N_{\phi} / \Delta_{\text {imp }}$ inside a LL and zero otherwise - Eq. (何) is changed into

$$
E_{n=0}=-\frac{2 N_{\phi}}{\Delta_{\text {imp }}} M^{2}=-\frac{2 N_{\phi}}{\Delta_{\text {imp }}} D^{2} \eta^{2},
$$

while Eq. (5) remains unchanged since it involves totally filled LLs. The electronic energy gain is now proportional to $\eta^{2}$. We therefore introduce still another effective elastic constant

$$
G^{\prime \prime}=G^{\prime}-2 \frac{N_{\phi}}{N_{p}} \frac{D^{2}}{\Delta_{\mathrm{imp}}}
$$

in order to write the total energy as $E_{\text {tot }}=N_{p} G^{\prime \prime} \eta^{2}$. The renormalized elastic constant $G^{\prime \prime}$ depends on the perpendicular magnetic field. A distortion only occurs if $G^{\prime \prime}<0$, i.e. $B_{\perp}>$ $B_{c} \equiv 2 \pi \hbar G^{\prime} \Delta_{\text {imp }} / 3 \sqrt{3} e a^{2} D^{2}$, which is always the case at large enough magnetic fields. This condition is precisely equivalent to requiring that the valley splitting $\Delta_{v}=2 D \eta$ - given by Eq. (9) with $\nu \approx 0$ - be larger than the LL width $\Delta_{\text {imp. }}$. Therefore, as soon as $B_{\perp}$ is larger than the threshold value $B_{c}$, the lattice is distorted - in other words, the ZO phonon is frozen-in - and the valley gap is larger than the LL width, which means that one can use the results obtained in the case of infinitely narrow LL. When $B_{\perp}<B_{c}$, the ZO phonon is strongly renormalized by a magnetic-field-dependent term and its frequency, in the adiabatic approximation, is

$$
\omega\left(B_{\perp}\right) \approx \omega(0)\left(1-\frac{B_{\perp}}{B_{c}}\right)^{1 / 2},
$$

where $\omega(0) \approx \omega_{0} \sqrt{1-\lambda}$ is the renormalized phonon frequency at zero magnetic field. The phase transition toward the CDW ground state occurs at $B_{\perp}=B_{c}$, where $\omega\left(B_{\perp}\right)=0$.

Here we exhibit a microscopic mechanism providing the coupling of the electrons to the ZO phonon - i.e. the non-zero deformation potential $D$ - via the interaction of the carbon atoms to the substrate. It is quite difficult to accurately predict the constant $D$ and we will 
therefore only provide an order of magnitude estimate. The mechanism that we think gives the largest contribution results from the interaction of a single carbon atom from the graphene sheet with the amorphous $\mathrm{SiO}_{2}$ substrate treated as a dielectric continuum. The non-retarded Lennard-Jones interaction energy of an atom at a distance $r$ of a dielectric wall is given by $E_{\mathrm{LJ}}(r) \approx-\left(\epsilon_{r}-1\right)\left\langle\mathbf{d}^{2}\right\rangle /\left(\epsilon_{r}+1\right) 48 \pi \epsilon_{0} r^{3}$, where $\left\langle\mathbf{d}^{2}\right\rangle$ is the atomic ground state expectation value of the squared electric dipole moment [20]. The on-site energy change resulting from the lattice distortion may be estimated as

$$
\pm M \approx E_{\mathrm{LJ}}\left(d_{0} \pm \eta\right)-E_{\mathrm{LJ}}\left(d_{0}\right) \approx \pm \frac{\epsilon_{r}-1}{\epsilon_{r}+1} \frac{\left\langle\mathbf{d}^{2}\right\rangle}{16 \pi \epsilon_{0} d_{0}^{4}} \eta
$$

where the \pm sign refers to sublattice $\mathrm{A}(+1)$ or $\mathrm{B}(-1), d_{0}$ is the average distance between the graphene sheet and the substrate and we assumed that $\eta \ll d_{0}$. For a carbon atom $\sqrt{\left\langle\mathbf{d}^{2}\right\rangle} \sim$ $4 e a_{0}$, where $a_{0}$ is the Bohr radius, which gives $D a \sim a\left(\epsilon_{r}-1\right) e^{2} a_{0}^{2} /\left(\epsilon_{r}+1\right) \pi \epsilon_{0} d_{0}^{4} \sim 1$ to $14 \mathrm{eV}$ depending on $d_{0} \sim 0.1$ to $0.2 \mathrm{~nm}$. Therefore, the order of magnitude of the deformation potential $D a$ is $5 \mathrm{eV}$. The condition $G^{\prime}>0$ that no Peierls instability occurs when $B_{\perp}=0$ implies that $D a<\sqrt{G a \hbar v_{F} / \gamma} \approx 9.8 \mathrm{eV}$. Therefore, in order to match the experiment [4], we take the plausible value $D a=7.8 \mathrm{eV}$, which gives $G^{\prime} a^{2} \approx 4.2 \mathrm{eV}$. In the end, the dimensionless electron-phonon coupling constant $\lambda=2 \gamma D^{2} /(3 G t) \approx 0.8$, which is quite a large value.

The mechanism proposed here features several differences with respect to the standard Peierls instability [17,19]: (i) the metal is two- rather than one-dimensional; (ii) the distortion does not change the Bravais lattice but only the point group symmetry - the inversion symmetry of the honeycomb crystal is broken by the instability but the triangular Bravais lattice is not affected by the distortion; (iii) the electron-phonon coupling does not exist in the isolated graphene crystal but is provided via the coupling to the substrate; (iv) the control parameter for the instability is the magnetic field rather than the temperature; (v) the metal (undoped graphene) is actually a zero gap semiconductor and has $k_{F}=0$ and the nesting condition is $|\mathbf{Q}|=0$, which indeed equals $2 k_{F}$.

\section{Charge density wave in graphene: direct electron-electron interactions}

Many authors have considered the effect of direct (Coulomb) electron-electron interactions in graphene, in particular to study the quantum Hall effect. Depending on which aspect of the Coulomb interaction is thought to dominate, different scenarios have been proposed. A popular scenario is that of quantum Hall valley and spin ferromagnetism, see e.g. 9, 21,22, 23, 24, This is the graphene version of the well-known quantum Hall ferromagnetism: the exchange interaction between electrons favors ferromagnetism inside a partially filled LL. Indeed, it allows one to reduce the interaction energy - in other words, to gain exchange energy - at no kinetic energy cost when the LL are assumed infinitely narrow and flat. This mechanism predicts plateaus at every integer filling factor in contradiction with the existing experiments. A possible way out of this contradiction is to consider strong disorder, which naively speaking, broadens the LL and therefore, by restoring the possibility of kinetic energy changes, works against ferromagnetism [21]. This could explain the absence of certain QH plateaus at magnetic fields up to $45 \mathrm{~T}$.

Apart from QH ferromagnetism, we are aware of only one other scenario: namely that of a commensurate A-B CDW ground state triggered by the magnetic field. This scenario exists in three different microscopic versions depending on which electron-electron interaction is believed to dominate. One version - the magnetic field induced Peierls instability - is the subject of the present paper and relies on electron-phonon coupling providing indirect interactions between electrons. The other two rely on Coulomb (direct) electron-electron interactions: one - the socalled magnetic catalysis - focusses on long-range Coulomb interactions, while the other relies on lattice-scale (extended Hubbard type) Coulomb interactions. All of these mechanisms lead to a similar ground state and therefore predict the same $\mathrm{QH}$ plateaus. 


\subsection{Long range Coulomb interactions}

In the context of highly oriented pyrolytic graphite, Khveshchenko suggested that long range Coulomb interactions could also lead to a CDW ground state in the presence of a magnetic field [6]. This idea stemmed from the phenomenon of dynamical chiral symmetry breaking originally proposed in relativistic theories of $(2+1)$-dimensional interacting Dirac fermions. In this scenario, the electron-electron interaction comes from the Coulomb interaction in the continuum limit in which only the long range part of the interaction survives. Within the CLL, electrons and holes pair up to form excitons, as a result of the attractive Coulomb interaction, and these excitonic pairs Bose condense. This leads to the creation of an excitonic gap in the electronic spectrum, which is similar to what we called the valley gap $\Delta_{v}$, but has a different magnetic-field dependence $\Delta_{\text {exciton }} \sim e^{2} /\left(\epsilon l_{B}\right) \propto{\sqrt{B_{\perp}}}^{4}$. This mechanism only occurs in the presence of a magnetic field and has therefore been dubbed the "magnetic catalysis" of the excitonic instability [6.7]. The ground state can be described either as a Bose condensate of excitons inside the CLL or as an A-B CDW.

\subsection{Lattice-scale Coulomb interactions}

Several years ago, in the context of STM studies on graphite surfaces, Tchougreeff and Hoffmann [25,26] suggested that graphene could have a CDW ground state ${ }^{5}$. They considered Coulomb interactions between electrons in an extended Hubbard model on the honeycomb lattice with on-site repulsion $U \sim e^{2} /(\epsilon a)$ and nearest-neighbor repulsion $V \sim U$, and neglected the longrange part of the Coulomb interaction. They showed that if the nearest-neighbor repulsion $V$ was strong enough to overcome the effect of the on-site repulsion $U$, a CDW ground state should be favored. In the opposite case, a spin density wave (SDW) would result. Recently, several authors have shown that a magnetic field should help to trigger this CDW instability [9.8] - or the SDW depending on the precise $U / V$ ratio [8]. In the CDW case, the corresponding valley gap is linear in the magnetic field $\Delta_{\text {Hubbard }} \sim U\left(a / l_{B}\right)^{2} \propto B_{\perp}$ just as in the magneticfield-dependent Peierls mechanism $\Delta_{v} \sim\left(D^{2} / G^{\prime}\right)\left(a / l_{B}\right)^{2}$.

\section{Conclusion}

We have reviewed the proposal of a magnetic-field-induced Peierls instability in graphene leading to a CDW ground state [5]. The driving mechanism is the electron - ZO phonon coupling, which is provided via the interaction of carbon atoms to the substrate. The instability corresponds to the spontaneous breaking of the inversion symmetry of the graphene honeycomb lattice and results in Dirac electrons having an effective mass proportional to the magnetic field. Apart from electron-phonon interactions, there are other interactions leading to a CDW instability triggered by a perpendicular magnetic field [6,7:9, 8]. As to the question of which is the dominant interaction leading to the instability in graphene, we mention two related examples of one-dimensional metals featuring a CDW instability. On the one hand, consider the well-studied case of polyacetylene, which dimerizes at low temperature and has long been described as being the prototypical example of a Peierls instability driven by electron-phonon interactions. It is now known that Coulomb interactions also play a very important role in the instability and that electron-phonon coupling alone is not enough to quantitatively explain the measured effect [28]. On the other hand, in a one-dimensional metal like KCP, the dominant mechanism leading to the Peierls distortion has been shown to be electron-phonon interactions [29]. In the case of graphene on substrate, it is therefore not clear at the moment whether a CDW does occur and if so, which is the driving mechanism leading to such a ground state in the presence of a magnetic field.

\footnotetext{
4 This excitonic gap is of the same order of magnitude as the LL spacing $\hbar \omega_{c}$ as the "graphene fine structure constant" $e^{2} /\left(4 \pi \epsilon \hbar v_{F}\right) \approx 0.5$.

5 Their work was actually triggered by an early experiment on a graphene sheet on the $\operatorname{Pt}(111)$ surface 27
} 


\section{Acknowledgments}

We thank M.O. Goerbig for useful discussions, and I. Herbut and D. Khveshchenko for comments on the manuscript.

\section{References}

1. K.S. Novoselov, A.K. Geim, S.V. Morozov, D. Jiang, M.I. Katsnelson, I.V. Grigorieva, S.V. Dubonos, and A.A. Firsov, Nature 438, (2005) 197.

2. Y. Zhang, Y.W. Tan, H.L. Stormer, and P. Kim, Nature 438, (2005) 201.

3. A.M.J. Schakel, Phys. Rev. D 43, 1428 (1991); Y. Zheng, T. Ando, Phys. Rev. B 65, 245420 (2002); V.P. Gusynin, S.G. Sharapov, Phys. Rev. Lett. 95, 146801 (2005); N.M.R. Peres, F. Guinea, A.H. Castro Neto, Phys. Rev. B 73, (2006) 125411.

4. Y. Zhang, Z. Jiang, J. P. Small, M. S. Purewal, Y.-W. Tan, M. Fazlollahi, J. D. Chudow, J. A. Jaszczak, H. L. Stormer, and P. Kim, Phys. Rev. Lett. 96, (2006) 136806.

5. J.N. Fuchs and P. Lederer, to appear in Phys. Rev. Lett.; arXiv:cond-mat/0607480.

6. D.V. Khveshchenko, Phys. Rev. Lett. 87, (2001) 206401.

7. V.P. Gusynin et al., Phys. Rev. B 74, (2006) 195429.

8. I. Herbut, arXiv:cond-mat/0610349; Phys. Rev. Lett. 97, (2006) 146401.

9. J. Alicea, M.P.A. Fisher, Phys. Rev. B 74, (2006) 075422.

10. P.R. Wallace, Phys. Rev. 71, (1947) 622.

11. R. Saito et al., Phys. Rev. B 46, (1992) 1804.

12. J.W. McClure, Phys. Rev. 104, (1956) 666.

13. R. Rammal, J. Physique 46, (1985) 1345.

14. G.W. Semenoff, Phys. Rev. Lett. 53, (1984) 2449.

15. F.D.M. Haldane, Phys. Rev. Lett. 61, (1988) 2015.

16. S. Piscanec, M. Lazzeri, F. Mauri, A.C. Ferrari, J. Robertson, Phys. Rev. Lett. 93, (2004) 185503.

17. R. Peierls, Quantum Theory of Solids (Oxford University Press 1955) pp. 108-112.

18. M.S. Dresselhaus, P.C. Eklund, Adv. Phys. 49, (2000) 705.

19. G. Grüner, Density Waves in Solids (Addison-Wesley Publishing Company 1994).

20. See e.g. A. Aspect, J. Dalibard, Séminaire Poincaré 1, (2002) 67.

21. K. Nomura, A.H. MacDonald, Phys. Rev. Lett. 96, (2006) 256602.

22. D.A. Abanin, P.A. Lee, L.S. Levitov, Phys. Rev. Lett. 96, (2006) 176803.

23. H.A. Fertig, L. Brey, Phys. Rev. Lett. 97, (2006) 116805.

24. M.O. Goerbig, R. Moessner, B. Douçot, Phys. Rev. B 74, (2006) 161407.

25. A.L. Tchougreef, R. Hoffmann, J. Phys. Chem. 96, (1992) 8993.

26. F.R. Wagner, M.B. Lepetit, J. Phys. Chem. 100, (1996) 11050.

27. T.A. Land, T. Michely, R. Behm, J.C. Hemminger, G. Comsa, Surf. Sci. 264, (1992) 261.

28. G. König, G. Stollhoff, Phys. Rev. Lett. 65, (1990) 1239.

29. R. Comès, M. Lambert, H. Launois, H. R. Zeller, Phys. Rev. B 8, (1973) 571. 